\title{
A Study on the Conceptual Design of Fashion Product
}

\author{
Yue Hu, Ying Liu \\ School of Fashion Engineering, Shanghai University of Engineering Science, Shanghai, China \\ Email: yorkhu@sues.edu.cn
}

How to cite this paper: $\mathrm{Hu}, \mathrm{Y} .$, \& Liu, Y. (2017). A Study on the Conceptual Design of Fashion Product. Art and Design Review, 5, 241-251.

https://doi.org/10.4236/adr.2017.54020

Received: September 14, 2017

Accepted: November 17, 2017

Published: November 22, 2017

Copyright $\odot 2017$ by authors and Scientific Research Publishing Inc. This work is licensed under the Creative Commons Attribution International License (CC BY 4.0).

http://creativecommons.org/licenses/by/4.0/

\begin{abstract}
In today's increasingly fashionable design society, the design of fashion products requires designers to have advanced consciousness and innovative spirit. Concept design is undoubtedly the most consistent with the development trend of the field of design theory. However, related studies are still scarce. This paper explains the definition of conceptual design and the conceptual design features of fashion products. Furthermore, some principles and methods of carrying out conceptual design of fashion products are analyzed preliminarily, with a view to further theoretical framework of fashion product conceptual design and its methods, to provide guidance for the development of design activities.
\end{abstract}

\section{Keywords}

Design Science, Fashion, Product, Conceptual Design, Design Concept

\section{Introduction}

In the article of Fashion Product Design Theory, the author primarily discusses the definition, attribute and category of fashion product with its design. It also puts forward that fashion product design is a creative comprehensive information process which shows up the form of products in a flat or three-dimensional form by using various "fashion elements". It is the process that converts people's pursuit of "fashion" or need to a specific physics or tools. At the same time, it is a method that designers are in the use of "fashion" to implement a planning, envisaging, and solving problems and through specific operations, expressing in the form of "fashion" (Hu, 2017).

Based on the above-mentioned basic principles, the "conceptual design" with its related issues of fashion product as well as the theory that enriches the fa- 
shion product design and its practice methods will be explained in this article, which aims to provide some new thinking and perspective for China's fashion design and education. If there is any omission, point out and correct it.

\section{The Interpretation of the Conceptual Design}

"Conceptual Design" was first proposed in the Conceptual Design written by American graphic designer Ellen Hobart Allen Hubert in the 1980s. The book contents how to organize ideas in graphic design, and how to endow design objects with a concept or idea for better promotion of enterprise as well as products and guiding consumers. At that time of the domestic design industry, very few people tried to understand the product design from point view of the "conceptual design" and cause enough attention.

Industrial design direction is the main force of the practice of "concept design" in the design field, because industrial products possess with mass, automatic and fashionable characteristics. The design will bring more direct impact on the production area which makes more rapid sensitive reaction to the market information. The conceptual design is particularly important before the promotion of a new product. Especially in automotive industry, the conceptual design becomes an engine that promotes the innovation of automotive industry. Car manufacturers often complete the exploration of new fashion, technology and function firstly through the conceptual design. At the same time, "concept car" has also become a benchmark leading development of automobile industry (Figure 1).

Based on the existing theoretical description, we can roughly define the conceptual design as follows:

First of all, it is a series of orderly, organized, targeted design activities that starts from analyzing consumers' needs to generating conceptual products. The

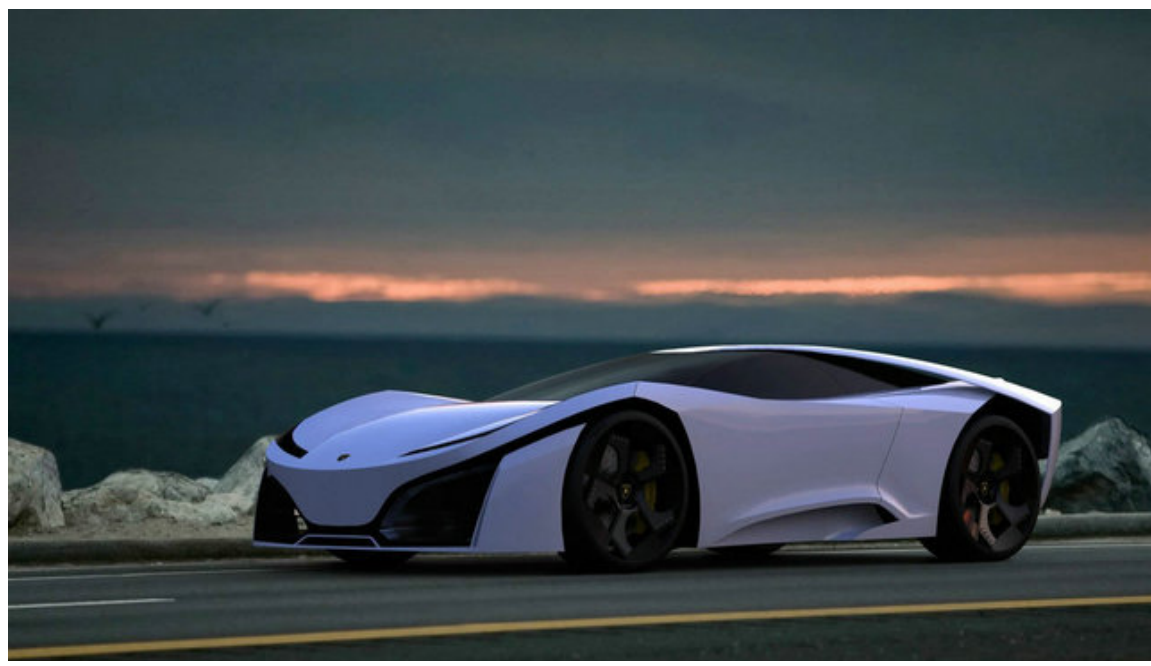

Figure 1. This Lamborghini hybrid concept car, called Madura, was designed by Slavche Tanevsky, a student of the Department of Science Applications at the University of Munich, Germany. 
development of a conceptual product needs to be established on the cornerstone of the target results through a large number of market research. To a large extent, the feasibility of this result determines the vitality of product after coming onto the market, so the basis of reliability is the key to success. And then it is a series of scientific rigorous design system engineering which ultimately derives the specific form, materials, structure and other content of abstract concept (Zhu, 2014).

Secondly, it is a process that is presented as a constantly evolving process from coarse to fine, from blur to clear, from abstract to concrete. In the early stages of the design, the designer must make a careful investigation and planning of the program to be designed, come out of a series of design ideas in which designer has to refine out the most accurate, the most feasible, the largest market potential conceptual model (Chen, 2013).

Thirdly, it is a design method that takes advantage of design concept which is set as a main line throughout the whole design process. Modern media and psychology firmly believe that concept is the conclusion of people's thinking about the characteristics and significance which can represent a certain thing or development process." The design concept is a summary of the thoughts generated from various emotional thinking summed up and refined by designers in view of the design (Ye, 2012). If the conceptual design is an article, then the design concept is the theme of this article. Conceptual design revolves around the design concept, and the design concept is linked to all aspects of conceptual design (Figure 2).

Finally, it is a way of thinking. It rises the designer's complex sensibility and instantaneous thinking to the unified rational thinking through design concept, which can help the designers successfully complete the whole design. Conceptual design is a goal that must be achieved through a reasonable path, with a basic logical framework. It is not a pure inspiration burst, not water without source or tree without roots, but a creative flash within highly rational thinking paradigm (Red Dot Design Concept Yearbook).

\section{The Main Features of Fashion Product Conceptual Design}

According to the interpretation of the conceptual design, we can clearly grasp the basic characteristics of fashion product conceptual design, and then build up theoretical system of such design so that guide the practice of design activities correctly.

\subsection{Creativeness}

Fashion product conceptual design firstly need to be creative, except for the basic measure of "fashion", the conceptual design needs more emphasis on the originality and original creativity of design, rejecting any existing things from form and content. Certainly, it doesn't mean that you can not use those symbols and material as well as procedures that have existed in history, but must apply to 


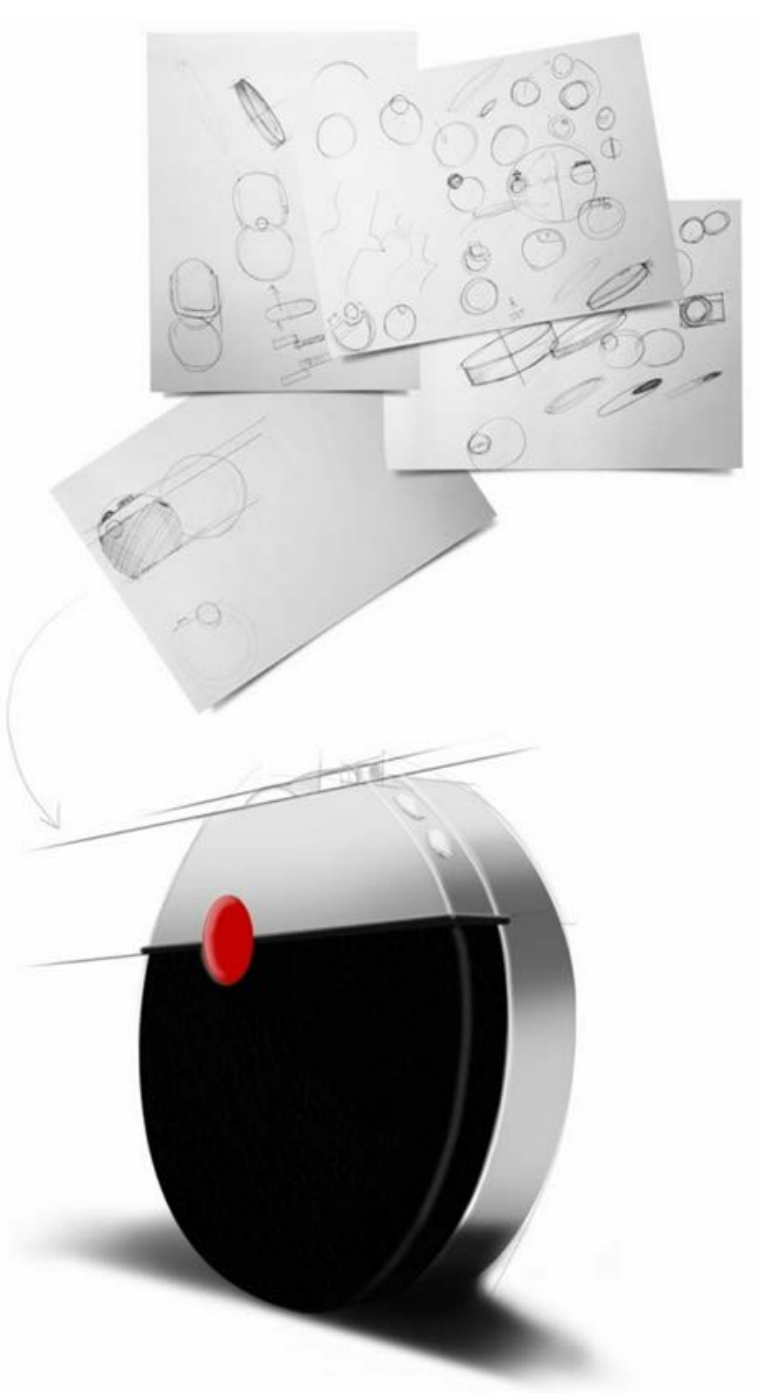

Figure 2. Swedish designer Vincent Sall proposed a subversive concept for the Leica X3, which inspired by the monochrome glasses.

practice in new ways and new perspectives. For example, the Bagigia brand launched a fashion handbag in the concept of hot-water bottle which was not original, when it was moved to the handbag, it has shown a particularly prominent original effect and fashion sense (Figure 3 ).

\subsection{Perceptiveness}

The conceptual design of fashion products can be not involved with many specific functional problems (such problems can be amended in the program design phase). As for functional issues, it can be conceptual, rational or logical inference, to put it more directly, just as "an armchair strategist". It is more like an exploratory scientific experiment, keeping a certain distance with the actual life so as to ensure enough imagination for thinking. For example, what will be the USB like in the future? How will storage technology develop? Now, come up with a concept known as the thinnest material "graphene" as the basis for the 


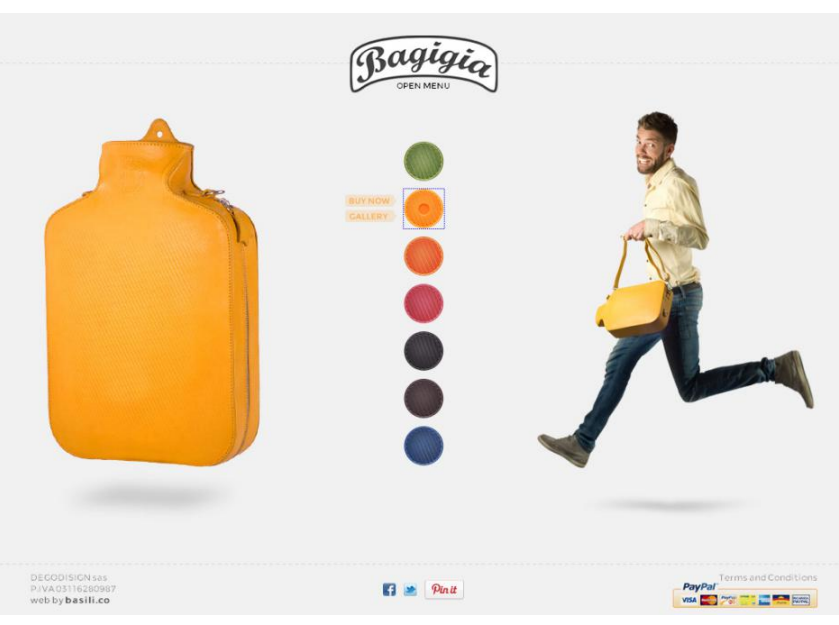

Figure 3. Bagigia brand launched fashion handbag products in the conceptual design of hot water bottle.

imagination, and the designer Aditi Singh \& Parag Anand proposed that based on this material, made it worthy of the name, thin as a note, also have a repeatable paste without leaving any traces. Paste it on the computer, television, music system when use it and then the device will be able to automatically read the data stored in them. And it also can be stacked to increase its capacity (Figure 4).

\subsection{Technological Features}

The conceptual design of fashion products requires us to be based on the most advanced technology and social awareness of the times, to have the courage to try out the latest things (new technology, new materials, new technical, new concept of life), to gather the most advanced technological achievements of the era, so that make it in the front of the times. Otherwise it is difficult to grasp the tide in the fashion trend, to lead the fashion or have chance to arouse the interest of the market. For example, the thing that looks like a watch is actually a fusion of high-tech conceptual mobile phones with flexible and transparent phone screen accommodated inside the a ring bracelet. Rollerphone, as for everyone, has a perfect fit of its own ergonomic design, which is customized for each user's measurement. It means that when the phone screen all stretched out, the top of the screen will be just contacted with your fingertips (Figure 5).

\subsection{Experimental Feature}

Any fashion concept has a certain degree of experimentation. It comes from some kind of concept and demand extracted from our needs in our daily life, or some kind of image that we want to advocate, propaganda and express. For example, the Chapelle de Ronchamp (designed by Le Corbusier) Fallingwater (designed by Wright), Farnsworth House (designed by Smith), etc., whether they have experienced what so-called the "conceptual design" stage or not, they are all presented the experimental characteristics of the conceptual design. The pioneers of these modern buildings have established their new architectural con- 


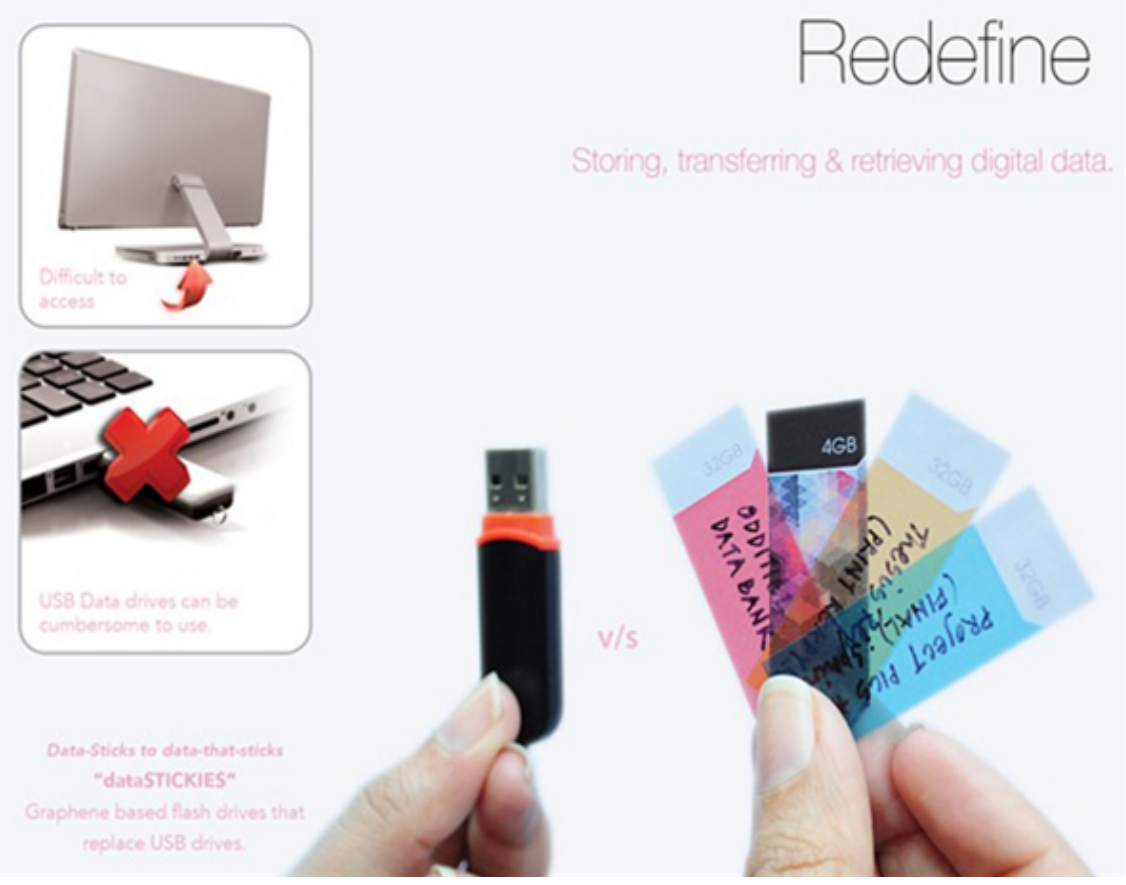

Figure 4. DataSticker storage put forward by Designers Aditi Singh \& Parag Anand.

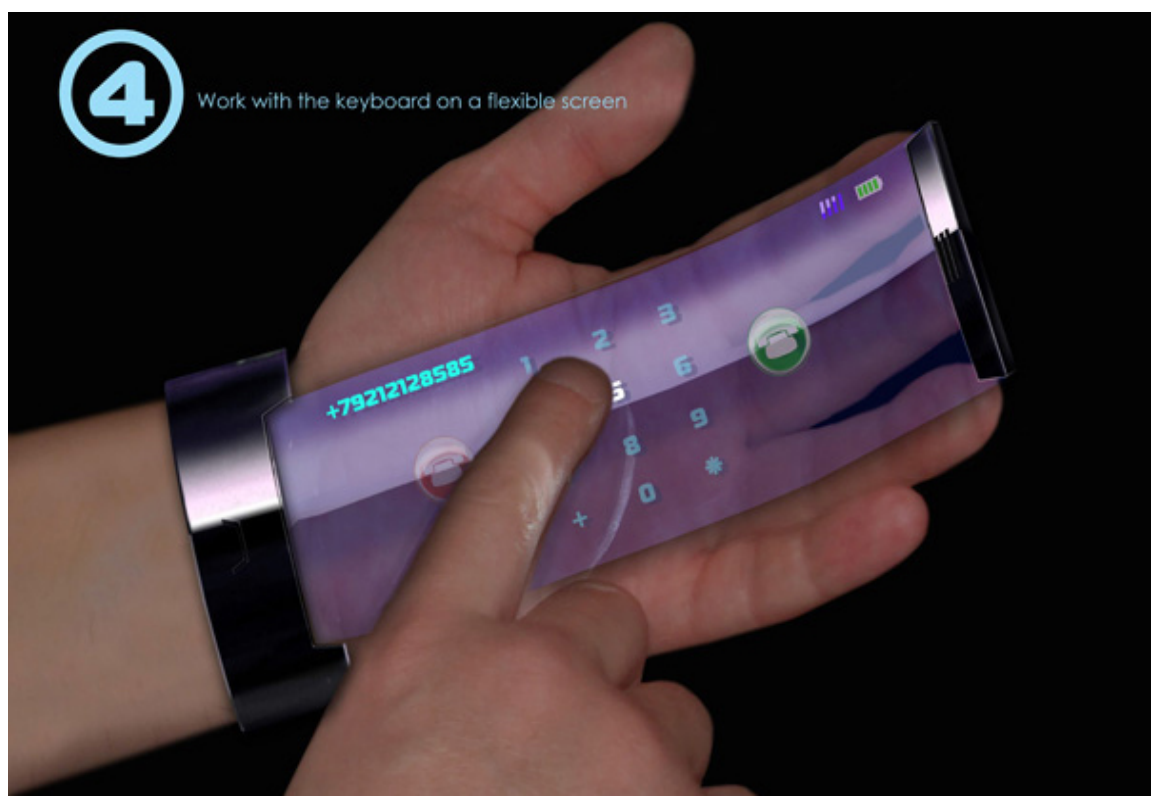

Figure 5. Conceptual Rollerphone.

cepts, without their unwavering practice of these ideas, their works can not have such a great influence on later generations.

\section{Principles of Fashion Product Conceptual Design}

Except for the above-mentioned characteristics, the conceptual design of fashion products are also required to meet one of the following four basic principles, or more, so that can live up to the essential attributes of contemporary design. 


\subsection{People Are Always the Core of the Design}

If green design related to environmental protection is the human's attention to nature, then the humanized design is the concern about people themselves. "People-oriented" design concept encourages designers to move the focus from product to product users-people, designing a more humanized products, which is the ultimate goal of fashionable designers. Although the digital age facilitates abundant social material, in this information explosion society, the network and the virtual community did not make the relationship between people more close, but strengthened the lifestyle of loneliness and privatization. The design thus carries more responsibility for the human spirit and spiritual comfort. For example, this is a digital product that may be applied to your home in the future. Flora is a digital conceptual vase designed by Japanese designer Yoshiki Matsuyama, allowing people to share flowers and greetings in a virtual way across geographical restrictions. Flowers placed in this vase can be copied through the Flora system into a virtual image sent to other places. Users can use 3D scanning cameras and projectors to design greetings and virtual flowers, so that help people's virtual living and communication more humanized in the future (Figure 6).

\subsection{Indispensable Stylish Atmosphere}

Fashion product conceptual design process is not only the process that designer give full play to the imagination via technology, but also a process which designers communicate with users to express users' fashion concept. People often want designers to design some characteristic products which can lead the fashion for them. Many companies are willing to produce daily necessities with strong stylish sense, such as Stark's juicer, Apple's electronic products, even if only a

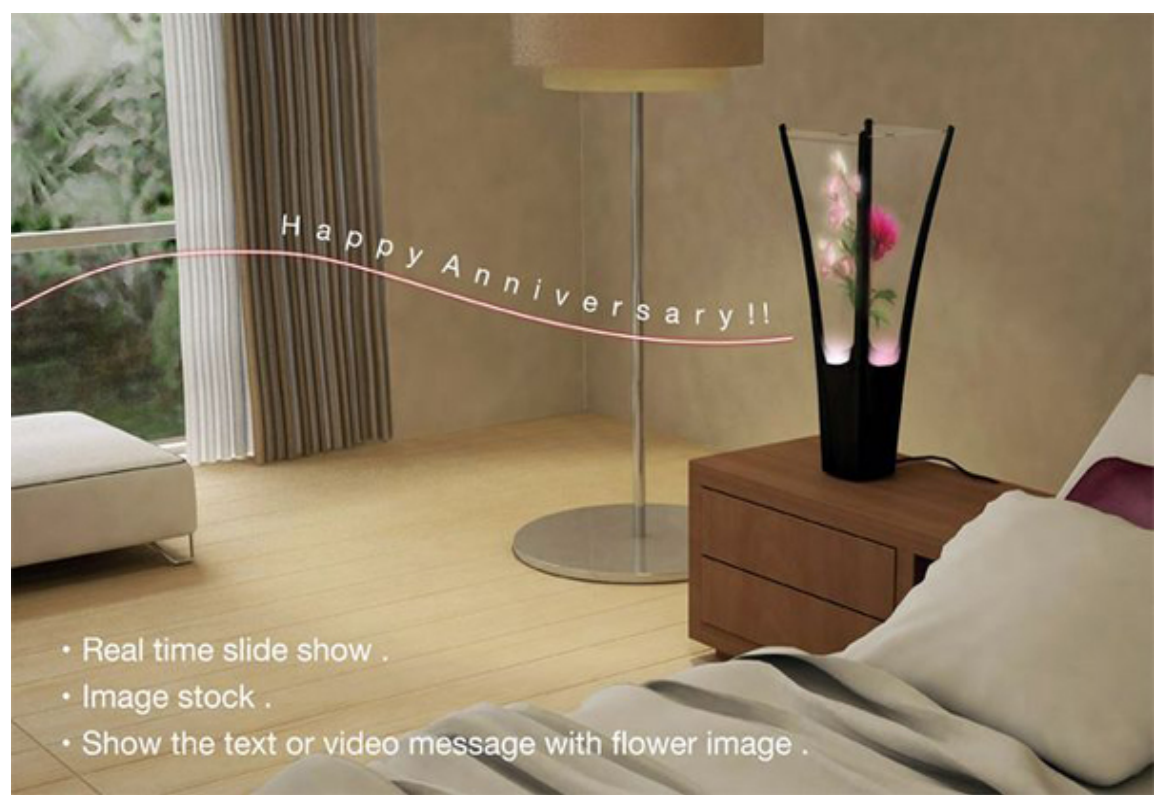

Figure 6. Digital conceptual vase designed by Japanese designer Yoshiki Matsuyama 
picture frame. IDEO believes that just as people have been free to change the protection of the computer screen pattern, in the future people want to customize products according to their own way, and the designer will take the fashion of product appearance and texture into consideration for the consumers. For example, this cool conceptual design-ring watch, a stainless steel ring on the cover of the three width of the outer ring with a scale which respectively, stands for the hours, minutes, seconds. These three outer ring in the continuous rotation, and white point highlighted is the current time (Figure 7).

\subsection{Indispensable Content of High-Technology}

Information age is the era in which science and technology are changing rapidly, and also a golden age when designers can give the vein to their creativity and imagination freely. If the designers' talent in the industrial era, have been bound by the technical constraints, then the contemporary technology development can be more powerful for the designer's imagination, but it requires designers to understand more about technology. The designer's design process increasingly requires the participation of technology, for example, how to coordinate the relationship between technology and users, how to make high-tech easier to use in the producing products become a new topic for designers. Many companies are increasingly relying on designers to produce high-tech fashion concept products which can guide the fashion trend, especially those products that roll the needs of consumers and the corresponding technology into one. The development of technology and the needs of enterprises encourage designers not only to design the form of product, but also to play a role of providing innovative products in the company. In the future, design will no longer be the designer's personal behavior, while cooperation with the technical staff will be the inevitable trend of the development of fashion product design.

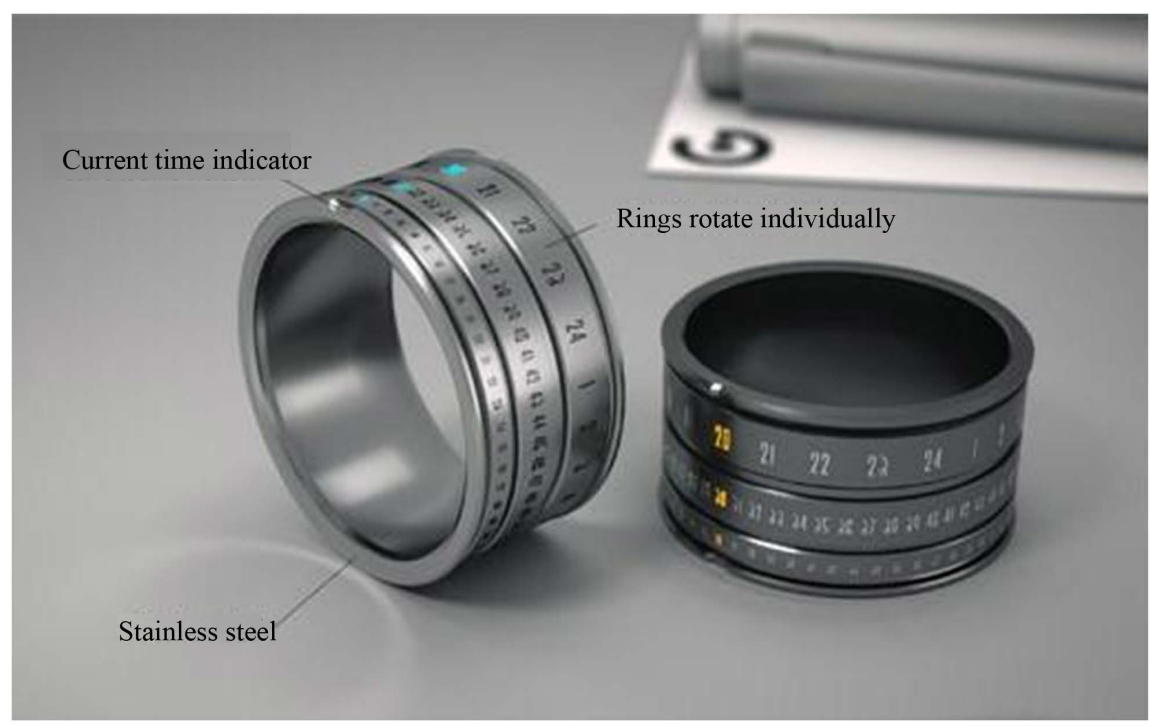

Figure 7. Conceptual design of ring watch with a strong sense of fashion. 


\subsection{Culture and Art Can Manifest the Humanistic Value}

Digital life has brought human beings into an unprecedented living state, and in the meantime the rapid development of technology make people feel out of control. Therefore, people need to recapture order, morality and ethics of human society through the history, culture and art without delay. It proves that technology can not create a better society, with the collapse of faith in industrialization and modernization, however, the continuation of culture is the most valuable things in human society. As the creators of lifestyle, our designs not only embodies the dual demand for material and spiritual, but also play an important role in human civilization. In the post-industrial era, the design has become a bridge between the technology and the humanistic culture. The lyrical characteristics and the expression of the poetic emotion have become the meaning of the fashion product concept design. People hope that the design can provide a better function revealing more sense of human and personality, and also hope that design can contain more humanistic value.

\section{The Conceptual Design Steps of Fashion Product}

\subsection{Clear Design Concept}

The determination of design concept is the key to the conceptual design of fashion product. Without a clear design concept, you will be lost in the trivial details. What is the purpose of your fashion product design? What kind of function does this fashion product have? What kind of need can this fashion product meet? These target question are the fundamental issues that should be determined at the beginning of the research phase of the design. After these questions meeting all the principles, you can fully release the creative inspiration so as to break through the realistic bottlenecks.

\subsection{The Formulation of Design Routes}

Fashion product conceptual design route is the way to achieve the above purpose fully expanding the imagination, and trying to achieve design concepts through a variety of path. For example, what product type will be designed? What kind of shape will be adopted? How to construct? What material will be used? What kind of function do they possess? What will be the way to achieve these goals and functions?

All the details, like all sizes of pearls scattered in the plate, which need linking into a stylish pearl necklace by designers' carding and series. It requires designers to have a logical organization and deductive thinking to coordinate the emotional creative thinking in this process.

\subsection{The Implementation of Design Expression}

Good design concepts and design routes still need to showcase through the best design expression. Without effective design expression, conceptual design of fa- 
shion product will become a pile of waste paper. The following four methods can be conceptual annotations placed in the "fashion product story version" (Figure 8).

\section{1) Hand-painted method}

The method which presented by the way of sketch or other hand-painted kinds.

\section{2) Digital method}

The method which assisted or totally presented by the computer program.

\section{3) Model method}

The method which presented by the model in actual or scaled size with some material.

\section{4) Text Instructed method}

The method which is instructed by texts to show the ideas etc.

\section{Conclusion}

At contemporary, with the design increasingly vogue, more and more advanced consciousness and innovative spirit are required in the design of fashion products. And conceptual design is undoubtedly the most consistent with the development of this new trend of design, while the relevant research is still deficient. This paper attempts to understand the definition of conceptual design and the conceptual design features of fashion products, and analyzes some principles and methods of carrying out the conceptual design of fashion products, and then constructs the theoretical framework of this design to guide the practice of design activities. The Fashion as a social phenomenon, represents the development of social culture. In the meantime, based on the unlimited business potential of the conceptual design of fashion product development, designers have realized practical significance of conceptual design on fashion product. Constrained by

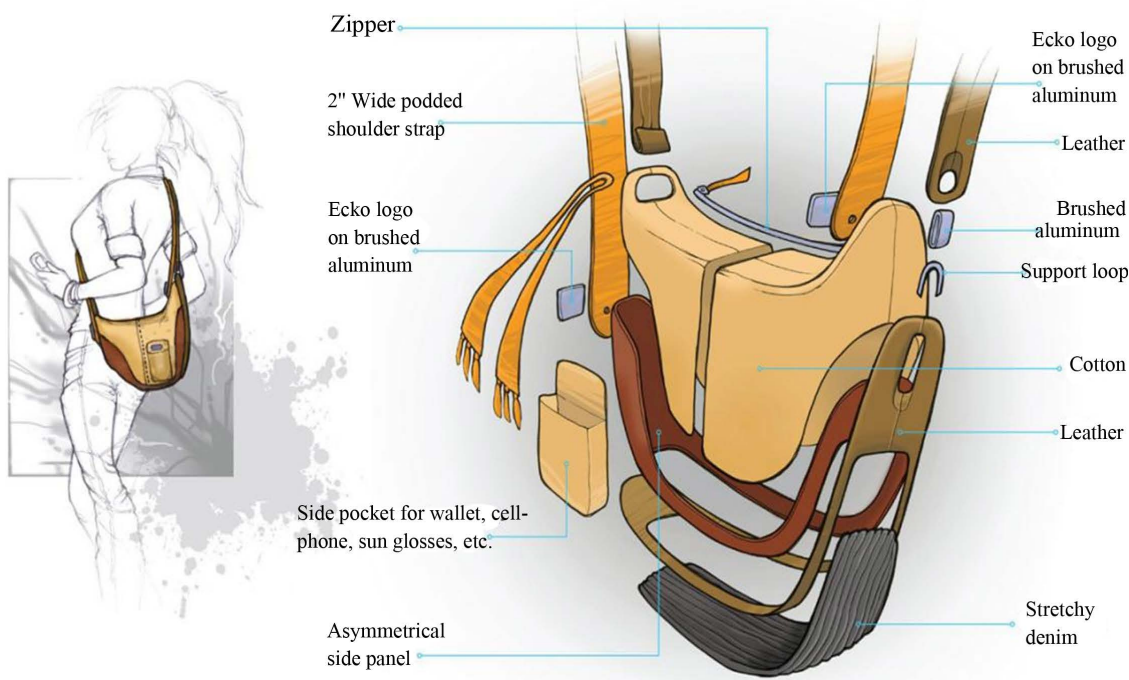

Figure 8. The story version that show the conceptual design of fashion product through the hand-painted, digital and copy method. 
space, this article for the concept of fashion product design is just a matter of course, hope to get your correction.

\section{References}

Chen, Y. (2013). On the Metaphor of "Design Concept" in Product Design. Packaging Engineering, 4, 23-31.

Hu, Y. (2017). Art Design Theory. Fashion Design and Engineering, 2, $22-27$.

Red Dot Design Concept Yearbook (2013/2014) Red Dot Award: Concept Design. Red Dot Editions (2014).

Ye, Q. (2012). Conceptual Design (pp. 128-135). Xi'an: China Construction Industry Press.

Zhu, X. L. (2014). "Fictional" Art-Product Conceptual Design. China Packaging Industry, 6, 47-52. 